\title{
Malformações congênitas em regiões de monocultivo no estado de Minas Gerais, Brasil
}

\author{
Congenital malformations in monoculture regions in the state of Minas Gerais, Brazil
}

Lidiane Silva Dutra $^{1}$, Aldo Pacheco Ferreira ${ }^{2}$

\begin{abstract}
RESUMO
Objetivo: analisar a associação entre o uso de agrotóxicos e as malformações congênitas em municípios com maior exposição, assim como avaliar a natureza da correlação existente entre a tendência observada e o volume de agrotóxicos considerados como disruptores endócrinos no estado de Minas Gerais, Brasil, entre 1994 e 2014. Modelo do Estudo: estudo transversal, de caráter exploratório, descritivo e quantitativo. Metodologia: foram analisadas as informações dos nascidos vivos (SINASC/ Ministério da Saúde), elaborando-se taxas de malformações ocorridas de 1994-2003 e 2004-2014. A associação entre os tipos de malformações e as variáveis foi testada pelo Odds Ratio para cada variável separadamente. A significância utilizada foi de $p<0,05$. Resultados: houve uma tendência crescente nas taxas de malformação congênita no estado de Minas Gerais, com destaque às regiões do Triângulo Mineiro/ Alto Paraíba e Vale do Mucuri/Jequitinhonha. Constatou-se que as taxas referentes às malformações congênitas foram maiores para os anos de maior exposição (2004-2014) e tiveram associação estatisticamente significativa para todas as malformações congênitas no estado de Minas Gerais. Conclusão: A exposição materna aos agrotóxicos foi relacionada à maior ocorrência de malformações congênitas.
\end{abstract}

Palavras-chaves: Malformações Congênitas. Agrotóxicos. Exposição Ambiental. Disruptores Endócrinos.

\begin{abstract}
Objective: to analyze the association between the use of agrochemicals and congenital malformations in municipalities with greater exposure, as well as to evaluate the nature of the correlation between the trend observed and the volume of agrochemicals considered as endocrine disruptors in the state of Minas Gerais, Brazil, between 1994 and 2014. Model Study: Cross-sectional study, exploratory, descriptive and quantitative character. Method: Rates of malformations for the periods 1994-2003 and 2004-2014 were calculated from the information on live births available at SINASC/Ministry of Health. Odds Ratio tested the association between types of malformations and variables for each variable separately. The significance was set at $p<0.05$. Results: there was an increasing trend in the rates of congenital malformation in the state of Minas Gerais, especially at Triângulo Mineiro/Alto Paraíba and
\end{abstract}

1. Doutoranda em Saúde Pública pela Escola Nacional de Saúde Pública Sergio Arouca (ENSP).

2. Professor Doutor da ENSP do Programa de Pós-Graduação em Saúde Pública.

Trabalho realizado no Programa de Pós-Graduação em Saúde Pública (ENSP).

Conflito de interesse: Os autores afirmam não haver nenhum conflito de interesse (financeiro e/ou pessoal) que possa afetar a veracidade deste manuscrito.
CoRRESPONDÊNCIA: Aldo Pacheco Ferreira Escola Nacional de Saúde Pública Sergio Arouca (ENSP). Rua Leopoldo Bulhões, 1480. CEP: 21041-210. Manguinhos. Rio de Janeiro (RJ). Brasil. 
Vale do Mucuri/Jequitinhonha regions. It was found that the rates for congenital malformations were higher for the years of greatest exposure (2004-2014) and had a statistically significant association for all congenital malformations in the state of Minas Gerais. Conclusion: Maternal exposure to pesticides was related with higher incidence of congenital malformations.

Keywords: Congenital Abnormalities. Agrochemicals. Environmental Exposure. Endocrine Disruptors.

\section{Introdução}

Define-se como malformação congênita (MFC) a anomalia funcional ou estrutural do desenvolvimento fetal decorrente de fator originado antes do nascimento, mesmo quando o defeito não for aparente no recém-nascido e só manifestar-se mais tarde. ${ }^{1}$ As MFC caracterizam-se em distúrbios de desenvolvimento presentes ao nascimento e que surgem no período embrionário e incluem toda alteração de ordem estrutural, funcional ou metabólica, que causam anomalias físicas ou mentais ao indivíduo. ${ }^{2}$ No Brasil, pesquisas realizadas pelo Estudo Colaborativo Latino Americano de Malformações Congênitas, evidenciaram taxa de 2,24\% a 5\% no nascimento de malformados, porém, em 2010, a prevalência de anomalias foi de $0,8 \%$ no país, representando média de dois mil nascimentos. ${ }^{3}$ Elas podem ser detectadas tanto no nascimento ou no decorrer da infância, a exemplo da estenose pilórica, a hérnia inguinal e algumas cardiopatias. ${ }^{4}$ Podem ser classificadas como isoladas ou associadas e de maior ou menor importância clínica. 3,5

Os processos de produção e consumo, baseados no crescimento e na globalização da economia mundial, vêm sendo criticados pelas ameaças à sustentabilidade ambiental e à saúde, sendo associados às iniquidades sociais que vulnerabilizam diferentes territórios e populações na sua condição de trabalhadores e moradores de áreas afetadas. ${ }^{6}$ Dados do Instituto Mineiro de Agropecuária - IMA (2015) comprovam que o estado de Minas Gerais ocupa o sexto lugar no comércio e no uso de agrotóxicos agrícolas no Brasil, atrás de Mato Grosso, São Paulo, Paraná, Rio Grande do Sul e Goiás.

A extensiva utilização de agrotóxicos representa um grave problema de saúde pública, principalmente nos países em desenvolvimento, cujas economias estão diretamente relacionadas aos produtos do agronegócio. ${ }^{7,8}$ Este modelo de desenvolvimento implica no uso crescente de aditivos quí- micos, o que justifica a preocupação acerca dos possíveis danos causados à saúde da população à médio e longo prazo. ${ }^{9,10,11}$

Existem mais de 18 mil produtos licenciados para uso, e a cada ano cerca de 1 bilhão de litros de agrotóxicos são aplicados na produção agrícola, residências, escolas, parques e florestas..$^{12}$ De acordo com a Agência Nacional de Vigilância Sanitária (Anvisa), o mercado mundial destes produtos cresceu $93 \%$ nos últimos dez anos, sendo que no Brasil, este crescimento chegou a $190 \% .^{13}$

Os agrotóxicos, largamente utilizados no mundo, constituem o maior grupo de substâncias classificadas como desreguladores endócrinos. ${ }^{14-18}$ $\mathrm{Na}$ classe dos agrotóxicos considerados desreguladores endócrinos estão inclusos inseticidas (deltametrina, carbofurano e organoclorados como DDT, DDE e lindano), herbicidas (atrazina, linuron e glifosato) e fungicidas (vinclozolina, penconazol, procloraz, promicida e tridemorfos) empregados na agricultura, aquicultura e uso domiciliar. ${ }^{7,10,19-25}$

No meio ambiente os agrotóxicos causam a contaminação do solo, poluição dos rios, além de persistirem nas cadeias tróficas. ${ }^{14,16,18,26} \mathrm{Em}$ relação à saúde humana, estes produtos podem provocar intoxicações (agudas ou crônicas), doenças como câncer, dermatoses, e muitas vezes até mesmo a morte. ${ }^{9,11,13,27}$

Os agrotóxicos com ação desreguladora endócrina podem ser encontrados nos alimentos, solo, água, vida selvagem e nos tecidos adiposos maternos, chegando às crianças por meio de exposições

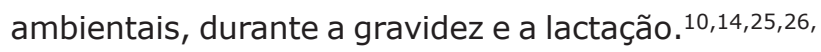
$28,29,30$ São capazes de afetar o sistema reprodutivo tanto de animais quanto de humanos, prejudicando o desenvolvimento embrio-fetal, o que pode resultar em MFC. ${ }^{26,27}$ As plantas e as culturas em geral podem absorver esses compostos diretamente da folhagem ou indiretamente por meio do solo, chegando aos seres humanos por meio da alimentação. ${ }^{10,22,31}$ 
As MFC afetam de $3-5 \%$ de todos os nascimentos, sendo que um terço destes defeitos põe em perigo a vida. ${ }^{32}$ Nos últimos tempos têm crescido o número de óbitos relacionados à $M C$, o que torna a prevenção e a investigação sobre os fatores de risco associados a esse desfecho de grande relevância para a saúde pública.33,34,35

Neste contexto, o presente estudo tem como objetivo analisar a distribuição temporal das MFC observadas no estado de Minas Gerais no período de 1994 a 2014, assim como avaliar a natureza da correlação existente entre a tendência observada e o volume de agrotóxicos considerados como disruptores endócrinos.

\section{Material e Métodos}

Trata-se de um estudo transversal, de caráter exploratório, descritivo e quantitativo, conduzido com informações dos nascidos vivos, sobre MFC ocorridas entre 1994 e 2014. As informações sobre os nascidos vivos foram obtidas do Sistema de Informações Sobre Nascidos Vivos (SINASC), Ministério da Saúde. ${ }^{36}$ Este é um sistema de informação de base populacional que agrega os registros contidos na declaração de nascidos vivos, o que permite diversas análises na área de saúde materno-infantil. Os anos escolhidos compõem todo o sistema do banco de dados, que começou os registros em 1994 e, até o presente momento, atualizou-os até 2014. Os indivíduos do estudo foram pareados segundo município e ano de ocorrência das MFC, procedendo-se ajuste na análise multivariada para controlar os fatores de confundimento. Foram desconsiderados os casos de nascidos vivos com registro ignorado ou desconhecido.

O tipo de MFC foi classificado de acordo com a Classificação Internacional de Doenças, $10^{\text {a }}$ revisão, (CID). ${ }^{37}$ Foi feita uma comparação entre o número de MFC ocorridas no estado de Minas Gerais, dividindo-se em duas partes o período acima mencionado: primeiro período (1994-2003) e segundo período (2004-2014). As ocorrências referentes ao primeiro período foram tidas como referência, uma vez que apresentavam um menor nível de exposição. Comparando-se os períodos assumidos, foram calculados O OR e o Intervalo de Confiança (IC) adotado para as amostras foi de $95 \%$. Também foram calculadas as taxas de MFC. ${ }^{38}$
As MFC também foram analisadas, de maneira mais específica, em três mesorregiões do Estado, das 12 estabelecidas pelo IBGE: Jequitinhonha (J), Vale do Mucuri (VM) e Triângulo Mineiro/Alto Paranaíba (TMAP), sendo que as duas primeiras foram agrupadas devido ao espaço geográfico por elas ocupado. ${ }^{39}$ Estas regiões foram escolhidas para representar, dentro do estado de Minas Gerais, diferentes exposições aos agrotóxicos, sendo uma de maior consumo (TMAP) e outra de menor consumo (JVM). Esta escolha foi feita com base nos dados obtidos pelo Instituto Mineiro de Agropecuária (IMA) e pelos mapas de consumo de agrotóxicos no Brasil. ${ }^{40}$

A relação dos agrotóxicos utilizados por princípio ativo para a média dos anos de 2013 a 2015 foi obtida através do IMA. Este instituto processa as informações recebidas através do Sistema Informatizado de Defesa Agropecuária (SIDAGRO), pelo qual as empresas comerciantes declaram as vendas destes insumos para os produtores mineiros.

Tendo como base a identificação de Mnif e colaboradores $^{30}$ elencando as 105 categorias de agrotóxicos e seus respectivos princípios ativos; para a presente pesquisa, dos 426 princípios ativos presentes na listagem do IMA, foram selecionados aqueles reconhecidos como disruptores endócrinos, resultando num total de 85 agrotóxicos. Dessa forma, para a construção da variável de exposição, além dos dados obtidos pelo IMA, levou-se em consideração a quantidade de lavouras plantadas por hectare, de acordo com o ano de plantio para cada região selecionada, como também para todo o estado de Minas Gerais (período de 1994-2014).

Selecionou-se 4 grãos de cultivo, principais commodites agrícolas brasileiras, que correspondem a mais de $60 \%$ da produção do estado, sendo eles algodão, cana-de-açúcar, milho e soja. Os dados sobre plantio foram obtidos através do Instituto Brasileiro de Geografia e Estatística. ${ }^{41}$ Também foram observados dados referentes ao consumo total de agrotóxicos no Estado entre 2000 e 2014, fornecidos pelo "Boletim de comercialização de agrotóxicos e afins" do Instituto Brasileiro do Meio Ambiente e dos Recursos Naturais Renováveis (IBAMA). ${ }^{42}$ Os dados referentes aos anos de 2006 e 2007 não estavam presentes no referido documento. Deste modo, foi feita uma média referente aos dois anos anteriores (2004 e 2005) para o ano de 2006 e dos dois anos posteriores (2008 e 2009) para o ano de 2007. 
Para a descrição dos resultados foram utilizadas as frequências relativas (percentuais) e absolutas ( $n$ ) das classes de cada variável qualitativa. As associações entre as variáveis independentes com a variável dependente foram estabelecidas. Valores de $p<0,05$ foram considerados significantes.

\section{Resultados e Discussão}

Comparando-se os dados detalhados obtidos no IMA e os dados brutos presentes no boletim de comercialização do IBAMA, percebe-se uma grande discrepância entre os números apresentados. A média de consumo para os anos de 2013-2014 seria de 34,2 mil toneladas, enquanto a média obtida através dos dados fornecidos pelo IMA, para 20132015, indicam um consumo médio de 2,3 mil toneladas. Os valores do IMA são 14,8 vezes menores que os do IBAMA, dado que pode ser indicativo de grande subnotificação por parte das empresas em Minas Gerais.

Houve maior número de MFC no período de 2003-2014, sendo que todas as associações entre exposição à agrotóxicos e MFC foram positivas e estatisticamente significativas. Para as mesorregiões, algumas das associações não foram estatisticamente significativas.

O Quadro 1 expressa a OR bruta e intervalos de confiança de nascidos vivos com MFC para todo o estado e para as mesorregiões analisadas, entre 1994-2014. Pode-se observar que as taxas de MFC para o período de 2004-2014 são 2,5 vezes maiores quando comparadas ao do período de 1994-2003, respectivamente 6,69 e 2,67. Este dado é alarmante, pois indica que em apenas uma década a incidência destas MFC dobrou.

A maior associação encontrada para as mesorregiões analisadas e a quarta para o Estado foi referente às "Malformações congênitas do aparelho circulatório", respectivamente: $\mathrm{OR}=2,79$, IC95\%= $1,87-4,16 ;$ OR $=3,27$, IC95\% $=2,83-3,77$. O estudo de Rappazzo e colaboradores ${ }^{43}$ demonstrou a associação para algumas MFC específicas cardíacas e níveis elevados de exposição à agrotóxicos. A "persistência do canal arterial", defeito congênito caracterizado pelo não fechamento do duto arterial após o nascimento, apresentou uma associação positiva e estatisticamente significativa com os índices mais elevados de exposição à agrotóxicos (maiores de 90\%), sendo estes índices de associação inexistentes em percentis de exposição menores $(O R=1,50$, IC95\%= 1,22 - 1,85). Já em um estudo caso-controle foi encontrada associação entre MFC cardíacas e a exposição ocupacional das mães a classes específicas de agrotóxicos, sendo estas respectivamente: defeito de septo atrial e inseticidas; síndrome da hipoplasia do coração esquerdo e inseticidas e herbicidas; tetralogia de Fallot e estenose da válvula pulmonar e fungicidas, herbicidas e inseticidas. ${ }^{44}$

A Tabela 1 apresenta alguns dos princípios ativos dos agrotóxicos considerados disruptores endócrinos, ${ }^{30}$ assim como as respectivas quantidades utilizadas em Minas Gerais e nas mesorregiões analisadas. Os valores, em quilos, correspondem à média de consumo entre os anos de 2013-2015. Esses princípios ativos selecionados representam $12,5 \%$ (287,32 toneladas) do total médio de todos os agrotóxicos consumidos em Minas Gerais (2.297,96 toneladas).

A Figura 1 apresenta a porcentagem dos sete princípios ativos mais utilizados no Estado e nas mesorregiões, juntamente com seus derivados e associações. A soma destes princípios ativos correspondem à mais de $90 \%$ do consumo na mesorregião do TMAP $(91,4 \%)$ e a mais de $80 \%$ na mesorregião do JVM $(88,0 \%)$ e no estado de Minas Gerais $(89,5 \%)$.

Foi identificado que os princípios ativos dos agrotóxicos considerados disruptores endócrinos, assim como a quantidade utilizada no estado de Minas Gerais e nas mesorregiões analisadas representam $12,5 \%$ ( 287,32 toneladas) do total médio de todos os agrotóxicos consumidos no estado (2.297,96 toneladas). Dessa forma, pode-se observar o maior quantitativo de uso de agrotóxicos na mesorregião do TMAP em relação à mesorregião do JVM. Comparando-se as mesorregiões como um todo, a porcentagem da mesorregião do TMAP corresponde a mais de $60 \%$ do consumo destes agrotóxicos $(63,43 \%)$ e a mesorregião do JVM pouco mais de $10 \%(11,09 \%)$. Embora a porcentagem da mesorregião do JVM seja pequena, os números absolutos são expressivos ( 31,9 toneladas), e se tornam ainda mais relevantes quando se dimensiona que estes agrotóxicos representam apenas uma parte do total de princípios ativos consumidos no Estado. 
Quadro 1: Odds Ratios de nascidos vivos com malformação congênita para variáveis controle em Minas Gerais e mesorregiões no período de 1994-2014.

\begin{tabular}{|c|c|c|c|c|c|c|c|c|}
\hline \multirow[t]{2}{*}{ Malformações Congênitas } & \multicolumn{4}{|c|}{ Minas Gerais } & \multicolumn{4}{|c|}{$\begin{array}{l}\text { Jequitinhonha/Vale do Mucuri } \\
\text { e Triângulo Mineiro/Alto Paranaíba, } \\
\text { 1994-2014 }\end{array}$} \\
\hline & 1994-2003 & 2004-2014 & OR & IC95\% & JVM & TMAP & OR & IC95\% \\
\hline Espinha bífida & 177 & 515 & 2,35 & $1,98-2,78$ & 20 & 53 & 1,50 & $0,90-2,51$ \\
\hline \multicolumn{9}{|l|}{ Outras malformações congênitas } \\
\hline do sistema nervoso & 834 & 1929 & 1,86 & $1,72-2,02$ & 207 & 276 & 0,75 & $0,63-0,90$ \\
\hline \multicolumn{9}{|l|}{ Malformações congênitas do } \\
\hline aparelho circulatório & 236 & 956 & 3,27 & $2,83-3,77$ & 29 & 143 & 2,79 & $1,87-4,16$ \\
\hline Fenda labial e fenda palatina & 484 & 1301 & 2,17 & $1,95-2,41$ & 78 & 226 & 1,64 & $1,27-2,12$ \\
\hline \multicolumn{9}{|l|}{ Ausência atresia e estenose } \\
\hline do intestino delgado & 7 & 45 & 5,18 & $2,34-11,49$ & 1 & 7 & 3,96 & $0,49-$ \\
\hline \multicolumn{9}{|l|}{32,21} \\
\hline \multicolumn{9}{|l|}{ Outras malformações congênitas } \\
\hline aparelho digestivo & 275 & 805 & 2,36 & $2,06-2,71$ & 55 & 152 & 1,56 & $1,15-2,13$ \\
\hline Testículo não-descido & 58 & 269 & 3,74 & $2,82-4,97$ & 54 & 39 & 0,41 & $0,27-0,62$ \\
\hline \multicolumn{9}{|l|}{ Outras malformações do } \\
\hline aparelho geniturinário & 467 & 1504 & 2,60 & $2,34-2,88$ & 92 & 264 & 1,62 & $1,28-2,06$ \\
\hline Deformidades congênitas do quadril & 23 & 64 & 2,24 & $1,39-3,61$ & 9 & 19 & 1,20 & $0,54-2,64$ \\
\hline Deformidades congênitas dos pés & 873 & 2525 & 2,33 & $2,16-2,52$ & 249 & 422 & 0,96 & $0,82-1,12$ \\
\hline \multicolumn{9}{|l|}{ Outras malformações e deformações } \\
\hline congênitas do aparelho osteomuscular & 1779 & 6276 & 2,84 & $2,70-3,00$ & 535 & 779 & 0,82 & $0,74-0,92$ \\
\hline Outras malformações congênitas & 558 & 2021 & 2,92 & $2,66-3,21$ & 160 & 323 & 1,14 & $0,95-1,38$ \\
\hline Anomalias cromossômicas NCOP* & 403 & 1075 & 2,15 & $1,92-2,41$ & 81 & 178 & 1,24 & $0,96-1,62$ \\
\hline Hemangioma e linfangioma & 43 & 90 & 1,69 & $1,17-2,43$ & 7 & 20 & 1,62 & $0,68-3,83$ \\
\hline \multicolumn{9}{|l|}{ Total de nascidos vivos com } \\
\hline malformações congênitas & 6217 & 19375 & 2,51 & $2,44-2,59$ & 1577 & 2901 & 1,04 & $0,98-1,11$ \\
\hline Taxa de MFC/1000 habitantes & 2,67 & 6,69 & & & 5,31 & 5,53 & & \\
\hline
\end{tabular}

Fonte: Instituto Mineiro de Agropecuária (IMA). Siglas: Triângulo Mineiro/Alto Paranaíba (TMAP), Jequitinhonha (J), Vale do Mucuri (VM), Minas Gerais (MG). *NCOP: não classificadas em outra parte; OR - Odds Ratio; IC - Intervalo de Confiança. 
Tabela 1: Princípios ativos referentes aos agrotóxicos utilizados em Minas Gerais, 2013-2015.

\begin{tabular}{|c|c|c|c|}
\hline \multirow[b]{2}{*}{ Agrotóxico (princípio ativo) } & \multicolumn{3}{|c|}{ Local aplicado (em Kg) } \\
\hline & TMAP & JVM & MG \\
\hline $2,4-d$ & $2.239 .855,34$ & $1.308 .685,30$ & $6.673 .321,17$ \\
\hline 2,4-d + aminopiralide & $3.905,24$ & $5.240,76$ & $12.343,05$ \\
\hline $2,4-d+$ picloram & $400.039,38$ & $2.608 .353,82$ & $4.553 .866,13$ \\
\hline 2,4-d, sal dimetilamina & - & - & 32,82 \\
\hline Acefato & $3.466 .421,68$ & $7.964 .223,80$ & $12.333 .583,43$ \\
\hline Alacloro & 415,67 & 1,33 & 778,67 \\
\hline Alacloro + trifluralina & 45,00 & 108,33 & 108,33 \\
\hline Aldicarbe & $1.566,67$ & $1.566,67$ & $1.566,67$ \\
\hline Atrazina & $10.808 .798,81$ & $1.587 .237,29$ & $22.299 .967,39$ \\
\hline Atrazina + alacloro & 2,46 & - & 331,28 \\
\hline Atrazina + simazina & $288.868,23$ & 521,50 & $1.057 .756,04$ \\
\hline Captana & $459.505,58$ & $230.162,09$ & $658.423,36$ \\
\hline Carbaril & 92,80 & 0,00 & 118,84 \\
\hline Carbendazim & $2.197 .389,49$ & $17.355,91$ & $4.361 .633,55$ \\
\hline Carbendazim + cresoxim-metílico + tebuconazol & $279.586,60$ & 58,01 & $371.825,93$ \\
\hline Carbendazim+tebuconazol & $114.143,48$ & 553,72 & $132.518,22$ \\
\hline Carbendazim+tiram & $62.934,15$ & $1.124,62$ & $126.944,55$ \\
\hline Carbofuran & $1.152 .801,00$ & $889.065,81$ & $1.979 .306,22$ \\
\hline Cimoxanil + clorotalonil & $509.772,46$ & $278.613,96$ & $830.031,60$ \\
\hline Cipermetrina & $219.453,44$ & $5.912,73$ & $313.050,02$ \\
\hline Cipermetrina+profenofós & 771,67 & 1,67 & $1.215,00$ \\
\hline Ciproconazol & $100.008,32$ & $2.571,16$ & $167.369,50$ \\
\hline Clorotalonil & $1.388 .158,92$ & $601.589,03$ & $3.342 .798,37$ \\
\hline Clorotalonil + cloreto de propamocarbe & - & - & 8,66 \\
\hline Clorotalonil+ oxicloreto de sódio & $25.229,79$ & $39.723,34$ & $43.615,14$ \\
\hline Clorotalonil + tiofanato metílico & - & - & - \\
\hline Clorpirifós & $3.389 .987,68$ & $139.446,52$ & $6.739 .074,14$ \\
\hline Deltametrina & $322.173,86$ & $427.504,78$ & $5.974 .304,75$ \\
\hline Deltametrina+triasofós & $5.455,00$ & $3.035,33$ & $24.017,33$ \\
\hline Dicofol & $76.214,82$ & 0,33 & $100.965,92$ \\
\hline Diflubenzurom & $88.322,97$ & $87.314,04$ & $156.015,25$ \\
\hline Dimetoato & $200.190,21$ & $2.108,03$ & $313.487,66$ \\
\hline Diurom & $888.361,96$ & $55.896,96$ & $1.350 .304,71$ \\
\hline Diurom + bromacil & $1.046,67$ & $1.206,67$ & $1.206,67$ \\
\hline Diurom + hexazinona & $314.461,90$ & $1.100 .512,55$ & $1.103 .696,03$ \\
\hline Diurom+hexazinona+sulfometurom metilico & $111.009 .511,47$ & $8.981,33$ & $12.161,36$ \\
\hline Diurom + tidiazurom & 14,80 & - & $111.001 .470,13$ \\
\hline Endosulfan & $49.265,31$ & 187,88 & $156.632,84$ \\
\hline Epoxiconazole & $22.834,65$ & $1.232,60$ & $125.974,82$ \\
\hline Epoxiconazole+cresoxim-metilico & $15.588,52$ & 298,67 & $51.659,97$ \\
\hline Fenarimol & 864,79 & $1.437,44$ & $32.032,23$ \\
\hline Fenitrotiona & 54,12 & 24,13 & $3.898,33$ \\
\hline
\end{tabular}


Fenitrotiona + esfenvalerate

Flutriafol

Flutriafol+carbendazim

Flutriafol+tiofanato metílico

Glifosato

Glifosato + imazetapir

Glifosato potássico

Glufosinato - sal de amonio

Glufosinato de amônio

Hexazinona + diurom

Iprodione

Linurom

Malationa

Methomil

Metomil + novalurom

Metribuzim

Paraquate+diurom

Parationa-metílica

Permetrina

Picloram $+2,4-d$

Piriproxifem

Procimidone

Procloraz

Propanil

Propiconazol

Propiconazol+ciproconazol

Propiconazol+difenoconazol

S-metocloro+ atrazina

S-metolacloro+glifosato

Tebuconazole

Tebuconazole+kresoxim-metilico

Tebuconazole+picoxistrobina

Tebuconazole+tridimenol

Tiametoxam + cipermetrina

Tiametoxam+ciproconazole

Tiofanato-metílico+clorotalonil

Triadimenol

Triadimenol+dissulfotom

Trifloxistrobin + cyproconazole

Trifloxistrobin + propiconazole

Trifloxistrobin+tebuconazol

Trifluralina
589,60

$313.863,07$

$14.360,75$

$1.680 .232,38$

$24.216 .338,07$

71,00

$8.845 .676,15$

$54.819,15$

$32.414,39$

$228.091,20$

$39.405,18$

$33.713,15$

$75.600,87$

$4.044 .042,77$

$395.881,20$

$63.818,07$

$141.569,18$

$5.112,24$

$10.289,60$

$20.822,72$

$182.333,28$

21,92

5,42

$118.364,07$

142,24

$50.515,38$

$202.486,03$

$10.504,67$

$291.563,28$

$14.117,08$

$339.572,92$

-

4,00

$321.855,43$

$24.735,93$

$2.743,33$

$76.939,58$

226.580,00

$47.397,27$
642,40

$38.535,01$

0,00

614,28

$12.787 .837,70$

$32.158,41$

363,85

$2.470,52$

$294.768,87$

$9.302,73$

$2.329,86$

$6.407,33$

$101.487,91$

$36.426,04$

$1.858,50$

$3.247,45$

$4.779,00$

$2.600,27$

$404.752,92$
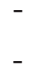

$4.330,45$

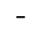

-

$1.530,53$

$1.038,43$

$9.943,00$

$2.347,83$

73,33

-

-

4,00

$724.977,92$

10,33

$10.641,33$

$5.228,71$

$7.255,89$

686,60
$1.888 .684,47$

$37.259,83$

$71.705,90$

$69.161 .887,15$

96,56

13.986.658,31

$96.166,71$

$95.598,66$

$300.539,88$

$62.516,71$

$69.517,44$

$335.857,09$

$9.168 .243,01$

18,92

$461.762,96$

$231.074,19$

$421.437,20$

$10.364,59$

$126.787,50$

$303.954,36$

$407.515,54$

142,94

382,50

$270.821,43$

205,72

$63.833,90$

$390.647,79$

$12.520,76$

$573.318,65$

$16.993,67$

$611.365,00$

112,50

-

4,00

$730.301,48$

$25.145,13$

$11.468,00$

$227.379,54$

18,53

$599.903,30$

$134.431,27$

$\begin{array}{ll}\text { Total } & 182.230 .707,44\end{array}$

$31.869 .867,15$

287.316.701,58

$\%$

63,43

11,09

100,00 


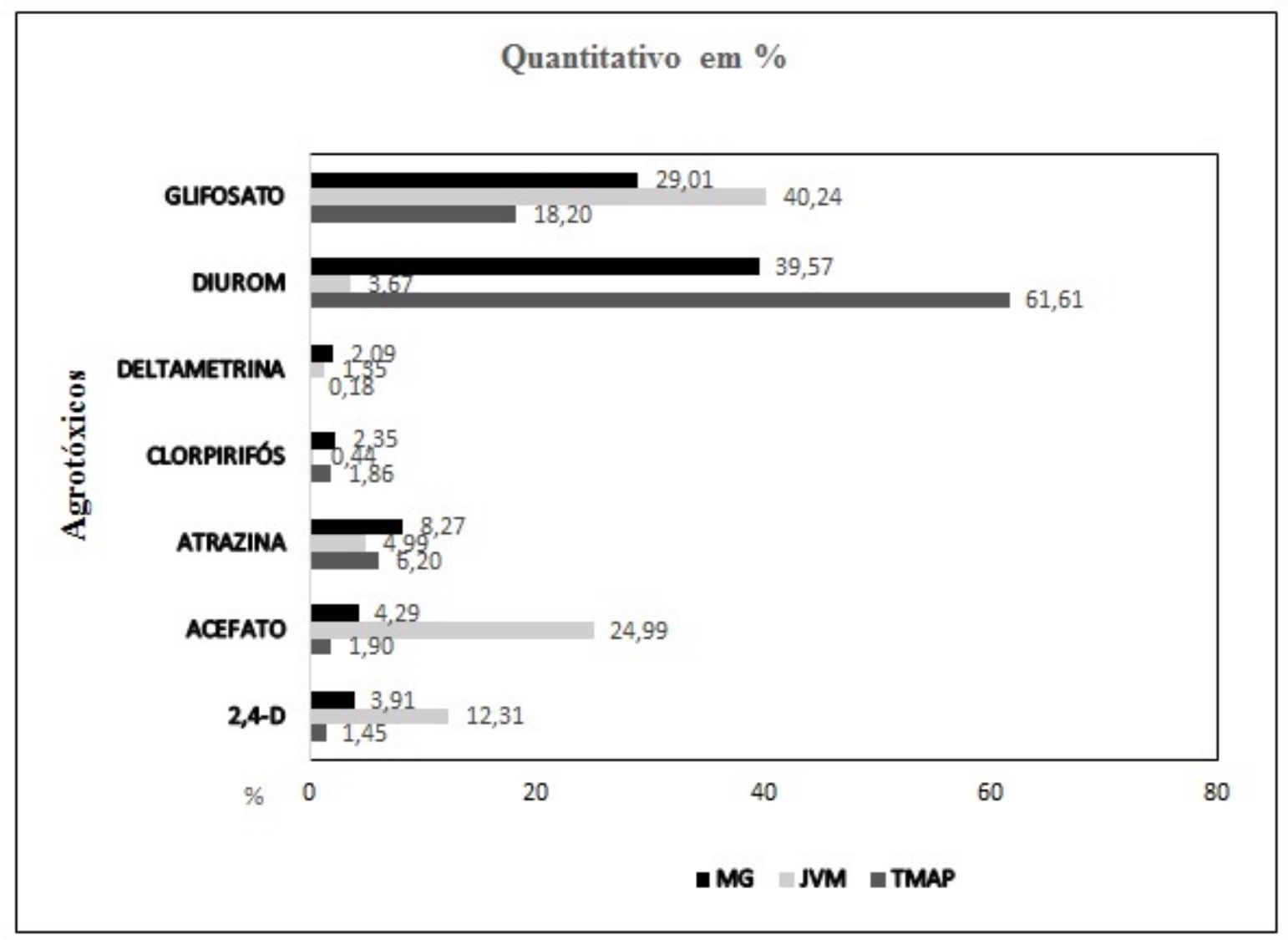

Figura 1: Percentual dos principais agrotóxicos utilizados em Minas Gerais, 2013-2015.

Fonte: Instituto Mineiro de Agropecuária (IMA). Siglas: Triângulo Mineiro/Alto Paranaíba (TMAP), Jequitinhonha (J), Vale do Mucuri (VM), Minas Gerais (MG).

A Figura 2 apresenta a área plantada referente aos principais grãos produzidos em Minas Gerais e nas mesorregiões analisadas, no período de 1994 a 2014, e o uso de agrotóxicos total entre 2000 e 2014. Entre 2000 e 2014 a área plantada para a produção de grãos aumentou em $62,2 \%(2,1$ milhões de hectares para 3,5 milhões de hectares) enquanto o consumo de agrotóxicos aumentou em $132,8 \%(14,4$ mil toneladas para 33,5 mil toneladas). ${ }^{41,42}$ Estes dados demonstram que a proporção entre área plantada e consumo de agrotóxicos é diferente, sendo esta última significativamente maior que a primeira. Corrobora, ainda, a hipótese de que a exposição ambiental sofrida pela população tem aumentado ao longo do tempo. Apesar do quantitativo de agrotóxicos não discriminar os princípios ativos utilizados, supõe-se que os mesmos não variaram significativamente, uma vez que as culturas de grãos continuam a ser as mesmas.
$\mathrm{Na}$ análise de um estudo caso-controle quanto a incidência de MFC cardíacas e a exposição ocupacional dos pais a substâncias químicas, embora não se tenha encontrado correlações significativas para a exposição ocupacional materna, a exposição paterna a ftalatos foi associada a uma maior incidência de defeitos congênitos cardíacos em geral $(\mathrm{OR}=2,08$; IC $95 \%=1,27-3,40)$, e especificamente a comunicação interventricular perimembranosa (OR $=2,84$; IC $95 \%=1,37-5,92)$. A exposição ocupacional paterna a compostos policlorados e aos alquilfenóis também foi associada, respectivamente, a: defeito do septo atrioventricular (OR $=4,22$; IC $95 \%=1,23-14,42$ ) e a coarctação da aorta $(\mathrm{OR}=3,85$; IC $95 \%=1,17-12,67) .45$

Em outro estudo caso-controle, foi analisada a incidência de diferentes tipos de malformações cardíacas e a exposição à agrotóxicos específicos devido à proximidade da residência dos indivíduos 


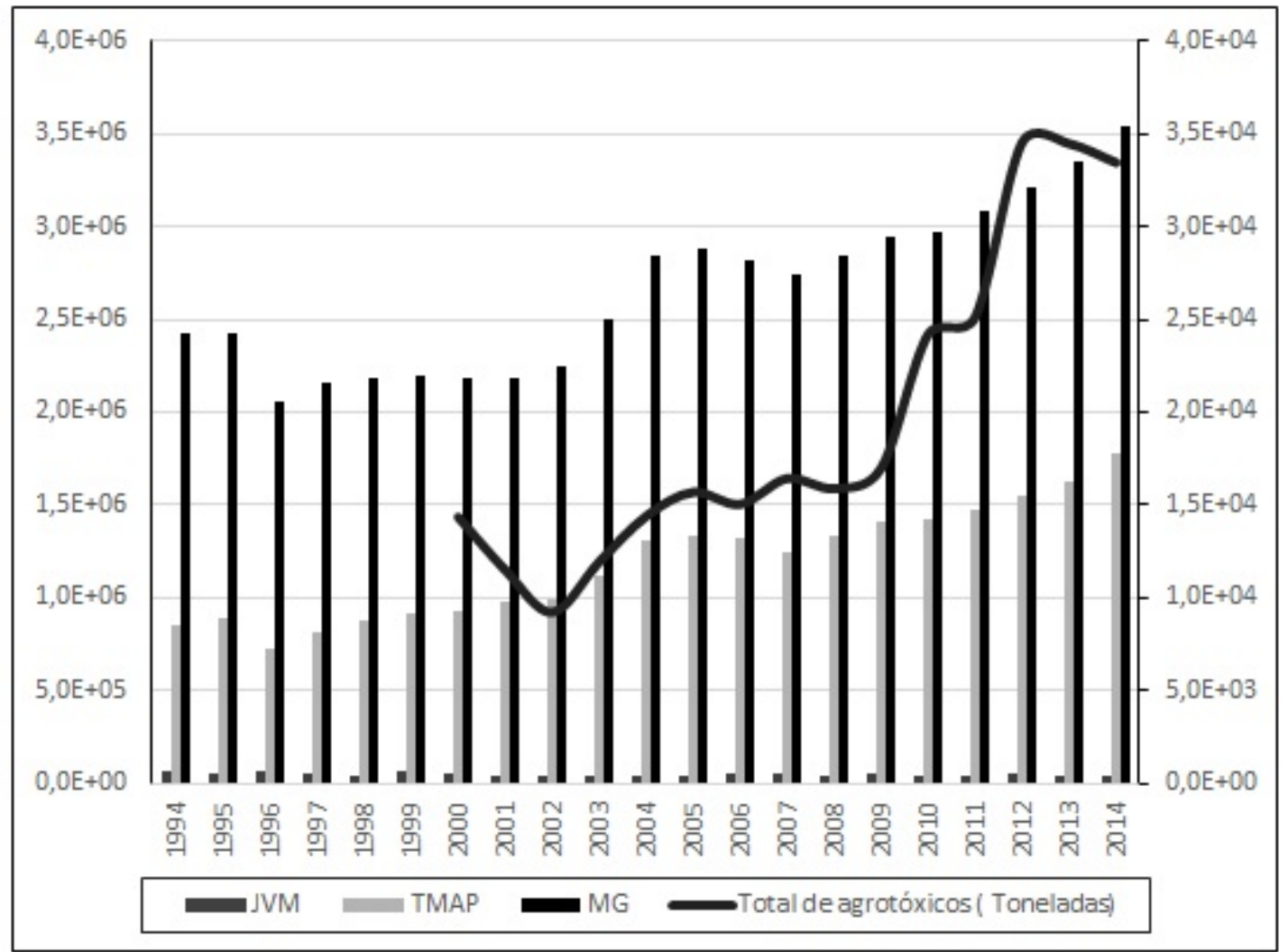

Figura 2: Relação entre área plantada e uso de agrotóxicos usados na produção de grãos em Minas Gerais e nas mesorregiões entre 1994-2014.

Fonte: Instituto Mineiro de Agropecuária (IMA). Siglas: Triângulo Mineiro/Alto Paranaíba (TMAP), Jequitinhonha (J), Vale do Mucuri (VM), Minas Gerais (MG).

a áreas de plantação. Foram encontradas associações entre MFC e agrotóxicos, respectivamente entre: tetralogia de Fallot e o neocotinóide imidacloprida; síndrome da hipoplasia do coração esquerdo e o fungicida azoxistrobina; estenose pulmonar valvar e os herbicidas norflurazon, 2,4-D e paraquat; defeito do septo ventricular perimembranoso e o acaricida abamectin; defeito de septo atrial e hexazinona, o herbicida 2,4-D, o acaricida óxido de fembutatina e os inseticidas clorpirifós e lambda-cialotrina. ${ }^{46}$

A segunda maior associação encontrada entre as mesorregiões analisadas foi referente às malformações de "Fenda Labial e fenda palatina" (OR $=1,64$, IC95\%= 1,27-2,12). Um estudo de meta-análise, que revisou publicações entre 19662005 , avaliou o risco de fendas orofaciais e a exposição a agrotóxicos. Foi encontrada associação positiva para a exposição ocupacional materna e o aumento do risco, ainda que moderado para fendas orofaciais $(\mathrm{OR}=1,37, \mathrm{IC}=1,04-1,81) .{ }^{47}$
Outro estudo destaca a relação entre agrotóxicos específicos e MFC específicas: anencefalia foi relacionada a 2,4-D, metomil, imidacloprida e ao éster fosfato $\alpha$-(para-nonil fenol)- $\omega$-hidroxi polioxietileno; espinha bífida a bromoxinil e a fenda labial e palatina a trifluralina e maneb. ${ }^{48}$

A terceira maior associação encontrada entre as mesorregiões analisadas foi referente à "Outras malformações do aparelho geniturinário" (OR $=1,62$, IC $95 \%=1,28-2,06)$, similar aos achados de Meyer e colaboradores ${ }^{49}$ que encontraram associação positiva entre a exposição à agrotóxicos específicos e hipospadia, um tipo de MFC do aparelho geniturinário masculino. Foram analisados 38 agrotóxicos que possuíam efeitos estrogênicos ou antiandrogênicos e outros com alguma evidência de toxicidade para a reprodução/desenvolvimento. A exposição ao herbicida diclofop-metil foi associada, significativamente, à hipospádia (OR $=1,08$; IC $95 \%$ $=1,01-1,15)$, sendo que o risco aumentou em $8 \%$ 
para os bebês de mães expostas quando comparados aqueles cujas mães não sofreram exposição.

A quarta maior associação encontrada entre as mesorregiões analisadas foi referente à "Outras malformações congênitas do aparelho digestivo" (OR $=1,56$, IC95\% $=1,15-2,13)$. Jiang et al. ${ }^{50}$ demonstraram a influência de alguns fatores na malformação congênita gastrointestinal, como ingestão de medicamentos, a ausência de ácido fólico, exposição a tintas e agrotóxicos. Dentre estas, a exposição materna a agrotóxicos antes ou durante a gravidez, teve a maior associação positiva encontrada $(\mathrm{OR}=15,20$, IC $95 \%=1,55-148,99)$.

Foi observada maior incidência de estenose hipertrófica congênita do piloro, um estreitamento da abertura entre o estômago e o intestino, nos casos relacionados aos percentis de maior exposição à pesticidas (50\% a $90 \%$ e acima de $90 \%)$, sendo as associações encontradas, respectivamente: $\mathrm{OR}=1,41$, IC95\% $=1,09-1,82 ;$ OR $=1,7$, IC95\% $=1,25-2,35$. Os dados observados se relacionam com os resultados obtidos por Rappazzo et al. ${ }^{43}$ no tocante as diversas MFC específicas e a exposição à agrotóxicos classificados em diferentes quantitativos.

Os resultados ora apresentados são decorrentes de um estudo transversal, exploratório, descritivo e quantitativo, conduzido com informações dos nascidos vivos, o que torna importante a realização de estudos longitudinais para estabelecer a relação de causalidade.

Quanto às limitações da forma de amostragem deve-se enfatizar que os sujeitos foram selecionados do SINASC. Este é um sistema de informação de base populacional que agrega os registros contidos na declaração de nascidos vivos, propiciando diversas análises na área de saúde maternoinfantil, favorecendo encontro de indivíduos com os desfechos de interesse do presente estudo. Como método de estudo, optou-se pelo método analítico, que permite estudar associações de forma exploratória e possibilita levantar hipóteses, porém não propicia o aprofundamento das questões abordadas.

Todos os dados apresentados corroboram a influência destes agrotóxicos nos desfechos analisados. Outro fato a ser considerado é o de que os agrotóxicos considerados teratogênicos também possuem diversos mecanismos de ação. Além de poderem atravessar a placenta e serem absorvidos sistemicamente, podem agir através da desrregulação endócrina, da indução ao dano genético, causando defeitos nas células neuronais e o estresse oxidativo, sendo estes os mecanismos propostos como principais para a toxicidade destes produtos perante o desenvolvimento. ${ }^{51} \mathrm{~A}$ atrazina, o alacloro e o clorpirifós são classificados como disruptores endócrinos, enquanto outros, como diuron e bifentrina, são classificados como substâncias tóxicas à reprodução. Levando-se em conta estes fatores, não se sabe quais as possibilidades de potencialização ou anulação dos efeitos quando existem tantos princípios ativos com mecanismos de ação e toxicologia diversos interagindo no corpo e no meio ambiente.

\section{Conclusão}

As taxas referentes às MFC foram maiores para os anos de maior exposição a agrotóxicos (2004-2014).-Nas últimas décadas, houve grande investimento das indústrias de agrotóxicos no comércio brasileiro, que foi potencializado devido a permissão de comercializar produtos banidos no exterior. Isto demonstra uma fraca política de vigilância ambiental sobre o consumo de agrotóxicos. Com efeito, há intensas pressões políticas e econômicas destas empresas sobre os órgãos regulamentadores brasileiros que influenciam as reavaliações dos produtos ao exercerem seu lobby em parlamentares e gestores, pressionando assim as políticas nacionais e dos estados. Outro fator agravante, neste contexto, é o subsídio governamental, seja através da obtenção de crédito rural facilitado para aqueles que adotam esse modelo de agricultura, seja pela isenção de impostos sobre o comércio de agrotóxicos, o que consequentemente aumenta o consumo dessas substâncias.

Muitas são as dificuldades no estabelecimento da relação entre MFC e a exposição a agrotóxicos, a despeito de se ter substâncias reconhecidamente disruptoras endócrinas presentes nesses químicos. Apesar de diversos entraves metodológicos, esses estudos subsidiam pressupostos acerca da exposição ambiental a esses contaminantes, e o aumento significativo nas taxas de MFC nos municípios que fazem uso de agrotóxicos corroboram essas hipóteses.

Além de todos os problemas já citados, o controle efetivo da comercialização destes produtos é 
muito pequeno no cenário brasileiro. Os dados referentes ao uso dos produtos não são sistematizados em bancos de dados informatizados para a grande maioria dos estados do país. Isto dificulta a mensuração do impacto da exposição ambiental destes produtos sofrida pela população. Soma-se a isto o lobby exercido pelas grandes corporações que impede, quase sempre, o acesso à informação. Apesar de diversos entraves metodológicos, estudos acerca do tema são importantes para alertar acerca dos problemas causados à saúde da população e para propor novas soluções perante o uso massivo destes produtos químicos.

\section{Referências}

1. Organização das Nações Unidas (ONU). Organização Mundial de Saúde (OMS). Organização Pan-Americana da Saúde (OPAS). Prevenção e controle de enfermidades genéticas e defeitos congênitos: relatório de um grupo de consulta. Washington DC: Organização Pan-Americana da Saúde (OPAS); 1984.

2. Horovitz DDG, Llerena Llerena Jr JC, Mattos RA. Atenção aos defeitos congênitos no Brasil: panorama atual. Cad. Saúde Pública. 2005;21:1055-64.

3. BRASIL. Ministério da Saúde. Secretaria de Vigilância em Saúde. Departamento de Análise de Situação de Saúde. Saúde Brasil 2011 : uma análise da situação de saúde e a vigilância da saúde da mulher. Brasília : Editora do Ministério da Saúde, 2012. 444 p.

4. Guerra FAR. Avaliação das informações sobre defeitos congênitos no município do Rio de Janeiro através do SINASC. [Tese de Doutorado]. Rio de Janeiro: Fundação Oswaldo Cruz; 2006.

5. Castro MLS, Cunha CJ, Moreira PB, Fernández RR, Garcias GL, Martino-Röth MG. Frequência das malformações múltiplas em recém-nascidos na cidade de Pelotas, Rio Grande do Sul, Brasil, e fatores sócio-demográficos associados. Cad. Saúde Pública. 2006; 22:1009-15.

6. Porto MF, Milanez B. Eixos de desenvolvimento econômico e geração de conflitos socioambientais no Brasil: desafios para a sustentabilidade e a justiça ambiental. Ciênc Saúde Coletiva. 2009; 14:1983-94.

7. Shaw Hughner R, Schultz Kleine S. Variations in lay health theories: implications for consumer health care decision making. Qual Health Res. 2008;18:1687-703.

8. Goldsmith P. The global view: issues affecting US production agriculture. J Agromedicine. 2010;15:184-91.

9. Pavão $A C$, Leão, MBC. Riscos de carcinogênese química no controle do Aedes. In: Augusto LGS, Carneiro RM, Martins $\mathrm{PH}$. Abordagem Ecossistêmica: Ensaios para o controle da dengue. Recife: Editora Universitária; 2005. p. 213-25.

10. Bedor CNG, Ramos LO, Pereira PJ, Rêgo MAV, Pavão AC, Augusto LGS. Vulnerabilidades e situações de riscos relacionados ao uso de agrotóxicos na fruticultura irrigada. Rev Bras Epidemiol. 2009;12:39-49.

11. Rosa IF, Pessoa VM, Rigotto RM. Introdução: agrotóxicos, saúde humana e os caminhos do estudo epidemiológico. In: Rigotto RM. (org). Agrotóxicos, Trabalho e Saúde vulnerabilidade e resistência no contexto da modernização agrícola no Baixo Jaguaribe/CE. Fortaleza/São Paulo: Edições UFC - Expressão Popular; 2011. p. 217-56.
12. Environmental Protection Agency. Promoting safety for America's Future: Office of Pesticide Programs FY 2002 Annual Report: 2003 EPA-735-R-03-001. Washington, DC: Environmental Protection Agency; 2003.

13. Cassal VB, Azevedo LF, Ferreira RP, Silva DG, Simão RS. Agrotóxicos: uma revisão de suas consequências para a saúde pública. REGET. 2014; 18:437-45.

14. Colucci MS, Bork H, Topp E. Persistence of estrogenic hormones in agricultural soils: I. 17â-Estradiol and Estrone. J Environ Qual. 2001; 30: 2070-6.

15. Birkett JW, Lester JN. Endocrine Disrupters in Wastewater and Sludge Treatment Processes. 1st ed. USA: IWA Publishing, Lewis Publishers CRC Press LLC, 2003.

16. Ghiselli G. Avaliação da qualidade das águas destinadas ao abastecimento público na região de Campinas: ocorrência e determinação dos interferentes endócrinos (IE) e produtos farmacêuticos e de higiene pessoal (PFHP). [Tese de Doutorado]. (Doutorado em Química Analítica) - Instituto de Química, Universidade Estadual de Campinas, Campinas; 2006.

17. Bila DM, Dezotti M. Desreguladores endócrinos no meio ambiente: efeitos e consequências. Quim Nova. 2007;30:651-66.

18. Américo JHP, Messias TG, Torres NH, Américo GHP. Desreguladores endócrinos no ambiente e seus efeitos na biota e saúde humana. Pesticidas: R Ecotoxicol e Meio Ambiente. $2012 ; 22: 17-34$.

19. Larini L. Toxicologia dos Praguicidas. São Paulo: Manole; 1999.

20. Maroni M, Colosio C, Ferioli A, Fait A. Organochlorine pesticides. Toxicology 2000;143:61-75.

21. Dores EFGC, De-Lamonica-Freire EM. Contaminação do ambiente aquático por pesticidas. Estudo de caso: águas usadas para consumo humano em Primavera do Leste, Mato Grosso - análise preliminar. Quim. Nova 2001;24: 27-36.

22. D'Amato C, Torres JPM, Malm O. DDT (dicloro difenil tricloroetano): toxicidade e contaminação ambiental - uma revisão. Quim Nova. 2002; 25:995-1002.

23. Ghiselli G, Jardim WF. Interferentes endócrinos no ambiente. Quim Nova. 2007;30:695-706.

24. Dores $\mathrm{EF}^{1}{ }^{1}$, Carbo L , Ribeiro ML , De-Lamonica-Freire EM. Pesticide levels in ground and surface waters of Primavera do Leste region, Mato Grosso, Brazil. J Chromatogr Sci. 2008;46:585-90.

25. Krauthacker B1, Votava-Raiæ A, Herceg Romaniæ S, Tjesiæ-Drinkoviæ D, Tjesiæ-Drinkoviæ D, Reiner E. Persistent organochlorine compounds in human milk collected in Croatia over two decades. Arch Environ Contam Toxicol. 2009;57:616-22.

26. Clementi M, Causin R, Marzocchi C, Mantovani A, Tenconi R. A study of the impact of agricultural pesticide use on the prevalence of birth defects in northeast Italy. Reprod Toxicol. 2007; 24:1-8.

27. Grisolia CK. Agrotóxicos - mutações, câncer e reprodução. Brasília: Universidade de Brasília; 2005.

28. Bathia R, Shiau R, Petreas M, Weintraub JM, Farhang L, Eskenazi B. Organocloride pesticides and male genital anomalies in the child health and development studies. Environ. Health Perspect. 2005;113:220-4.

29. Kidd KA, Blanchfield PJ, Mills KH, Palace VP, Evans RE, Lazorchak JM, Flick RW. Collapse of a fish population after exposure to a synthetic estrogen. Proc Natl Acad Sci. 2007; 104:8897-901.

30. Mnif W, Hassine AIH, Bouaziz A, Bartegi A, Thomas O, Roig B. Effect of Endocrine Disruptor Pesticides: A Review. Int J Environ Res Public Health. 2011;8:2265-303. 
31. Rigotto RM, Silva AMC, Ferreira MJM, Rosa IF, Aguiar ACP. Tendências de agravos crônicos à saúde associados a agrotóxicos em região de fruticultura no Ceará, Brasil. Rev Bras Epidemiol 2013; 16: 763-73.

32. Benítez-Leite S, Macchi ML, Acosta M. Malformaciones congénitas associadas a agrotóxicos. Arch Pediatr Urug 2009; 80: 237-47.

33. García AM, Fletcher T, Benavides FG, Orts E. Parental agricultural work and selected congenital malformations. Amer. J. Epidemiol 1999;149:64-74.

34. Gandolfi F, Pocar P, Brevini TAL, Fischer B. Impact of endocrine disrupters on ovarian function and embryonic development. Domest Anim Endocrinol. 2002;23:189-201.

35. Inadera $\mathrm{H}$. The immune system as a target for environmental chemicals: xenoestrogens and other compounds. Toxicol Lett. 2006;164:191-206.

36. Ministério da Saúde. DATASUS. Sistema de Informação de Nascidos Vivos. Disponível em: http://tabnet.datasus. gov.br/cgi/deftohtm.exe?sinasc/cnv/nvCE.def [Acesso em 13 de maio de 2016].

37. Armitage $P$, Berry G, Matthews JNS. Statistical Methods in Medical Research. 4th Edition. Wiley-Blackwell; 2001.

38. CID (Classificação Estatística Internacional de Doenças e Problemas Relacionados à Saúde). $10^{a}$ revisão. São PauIo: Centro Colaborador da OMS para a Classificação de Doenças em Português, Universidade de São Paulo; 1994.

39. Medronho RA, Carvalho DM, Bloch KV, Luiz RR, Werneck GL. Epidemiologia. 2a ed. São Paulo: Atheneu; 2005.

40. Minas Gerais. Governo do Estado. Divisão de Minas Gerais em Mesorregiões e microrregiões (IBGE). Disponível em: https://www.mgweb.mg.gov.br/governomg/portal/c/ governomg/conheca-minas/geografia/5669-localizacaogeografica/69547-mesorregioes-e-microrregioes-ibge/ 5146/5044 [Acesso em 25 de maio de 2016].

41. Brasil. Instituto Brasileiro de Geografia e Estatística. Produção Agrícola Municipal. Disponível em: http:// www.sidra.ibge.gov.br/bda/tabela/listabl.asp?c=1612\&z= t\&o=11 [Acesso em 05 de abril de 2016]

42. Bombardi LM. Pequeno Ensaio Cartográfico Sobre o Uso de Agrotóxicos no Brasil. São Paulo: Laboratório de Geografia Agrária. USP; 2016: p. 10.
43. Rappazzo KM, Warren JL, Meyer RE, Herring AH, Sanders AP, Brownstein NC, Luben TJ. Maternal residential exposure to agricultural pesticides and birth defects in a 2003 to 2005 North Carolina birth cohort. Birth Defects Res A Clin Mol Teratol. 2016;106:240-9.

44. Rocheleau CM, Bertke SJ, Lawson CC, Romitti PA, Sanderson WT, Malik $S$, et al. Maternal occupational pesticide exposure and risk of congenital heart defects in the $\mathrm{Na}$ tional Birth Defects Prevention Study. Birth Defects Res A Clin Mol Teratol. 2015;103:823-33.

45. Snijder CA, Vlot IJ, Burdorf A, Obermann-Borst SA, Helbing WA, Wildhagen MF, et al. Congenital heart defects and parental occupational exposure to chemicals. Hum Reprod. 2012;27:1510-7.

46. Carmichael SL, Yang W, Roberts E, Kegley SE, Padula AM, English PB, et al. Residential agricultural pesticide exposures and risk of selected congenital heart defects among offspring in the San Joaquin Valley of California. Environ Res. 2014;135:133-8.

47. Romitti PA, Herring AM, Dennis LK, Wong-Gibbons DL. Meta-analysis: pesticides and orofacial clefts. Cleft Palate Craniofac J. 2007;44:358-65.

48. Yang W, Carmichael SL, Roberts EM, Kegley SE, Padula AM, English PB, Shaw GM. Residential agricultural pesticide exposures and risk of neural tube defects and orofacial clefts among offspring in the San Joaquin Valley of California. Am J Epidemiol. 2014;179:740-8.

49. Meyer KJ, Reif JS, Veeramachaneni DN, Luben TJ, Mosley BS, Nuckols JR. Agricultural Pesticide Use and Hypospadias in Eastern Arkansas. Environ Health Perspect. 2006; 114:1589-95.

50. Jiang X, Xu G, Shen L, Wu J, Chen H, Wang Y. Influential factors on congenital gastrointestinal malformation: a hospital-based case-control study. Zhonghua Liu Xing Bing Xue Za Zhi. 2014;35:81-4.

51. van Gelder MM, van Rooij IA, Miller RK, Zielhuis GA, de Jong-van den Berg LT, Roeleveld N. Teratogenic mechanisms of medical drugs. Hum Reprod Update. 2010;16:378-94. 